Proceedings

\title{
Gamma Radiation Induces Apoptosis by Over Expression of PERP Effector of p53-dependent Cell Death in HeLa Cells ${ }^{+}$
}

\author{
Walid Mahmoud Khalilia 1,* and Gül Özcan ${ }^{2}$ \\ 1 Department of Forensic Sciences, Al-Istiqlal University, Jericho, Palestine \\ 2 Faculty of Science, Biology, Istanbul University, 34126 Istanbul, Turkey; gozcan@istanbul.edu.tr \\ * Correspondence: khaliliawalid@gmail.com; Tel.: +970-598515660 \\ $\dagger$ Presented at the 1st International Electronic Conference on Cancers: Exploiting Cancer Vulnerability by \\ Targeting the DNA Damage Response, 01-14 February 2021, Online.
}

Received: date; Accepted: date; Published: date

\begin{abstract}
The objectives of this study were to determine the configuration of several genes in response to Gamma Radiation (GR) treatment. Novel treatment strategy to increase the cancer cell sensitivity to radiotherapy by modulation of the PERP expression could be developed for cervical cancers. Cervical cancer cells were incubated for several periods after were exposed to various doses of GR, MTT assay were used to explore propagation of HeLa cells, apoptotic Index (AI) were measured using fluorescent microscopy by estimating apoptotic morphological features. While, signalling pathway analyses were performed on up-regulated genes which evaluated using microarray technology. From the results of this study, the proliferation of HeLa cervical cancer cells exposed to GR was inhibited proportionally with dose and time after exposure. Also, apoptotic morphological features, such as shrinkage of the cell and formation of apoptotic bodies, was clearly visible under the microscope for irradiated HeLa cells. after $48 \mathrm{~h}$. exposure to different doses of GR, the dose of 32 Gy was specified as an AI dose. The mRNA levels of pro-apoptotic genes such as, PERP; BAX; CASP9; TRAF3 and other factors detected by microarray after treatment with GR were up-regulated. Whereas, many anti-apoptosis factors were down-regulated. P53 pathway were significantly reinforced after pathway analysis for up- and dawn- regulated genes. For conclusion, GR induces apoptosis by over expression of PERP factor and p53-dependent cell death in cervical carcinoma HeLa cells.
\end{abstract}

Keywords: Apoptosis; Ionizing Radiation; HeLa cells; PERP; Microarray; MTT assay; DAPI staining

\section{Introduction}

GR, leads to a variety of cellular damages including nucleic acids and membrane hurt. DNA damage leads to a coordinate network of signal transduction pathways involved in cell cycle arrest, DNA repair and the activation of apoptosis processes. Apoptosis is the major mode of programmed cell death, and is characterized by a series of morphological hallmarks, including cell shrinkage, DNA condensation and fragmentation, followed by the formation of apoptotic bodies [1].

Ionized radiation leads to a modulation of the expression of many genes that control complex regulatory pathways. Identification of specific genes may allow the determination of pathways important in radiation responses. In replay to GR exposure, several signal transduction pathways, such as ERK1/2, JNK, p38, and ATM and transcription factors, such as AP1, NF $\kappa$ B, GADD153, and p53 are activated. tumor suppressor $\mathrm{p} 53$ gene, which regulates apoptosis under stress conditions like hypoxia, ionized radiation and free radical formation initiate p53-mediated DNA repair and apoptosis. p53 essential function controls the transcription of many other genes. When DNA damage 
develops for any reason, the normally inactive tumor suppressor p53 gene activates, which stimulates the p21 gene which leads to DNA repair and apoptosis. p53-mediated apoptosis induced by oncogene activation, such as BAX, and c-MYC $[2,3]$. Another apoptotic gene, PERP, is induced in response to DNA-damage. PERP is a transmembrane protein that represents a new member of the PMP-22/Gas family of proteins implicated in cell growth regulation. The kinetics of PERP induction in response to DNA damage and the presence of a p53-responsive element in the PERP promoter support the concept that it is a direct p53 target. The mechanism by which PERP contributes to p53-mediated apoptosis is yet to be defined. On the other hand, a sequence similarity between PERP and the calcium channel suggests that it could have channel activity, maybe allowing some critical molecule significant for activating apoptosis to pass through. However, PERP may lastly act by affecting regulators of the apoptotic processes, such as Bcl2 and BAX, or by directly activating apoptotic effectors like Caspases [4-6]. Therefore, the aims of this study were to determine the expressing of many genes in response to GR processing. Novel treatment strategy to increase the cancer cell sensitivity to radiotherapy by modulation of the PERP expression could be developed.

\section{Materials and Methods}

Cervical adenocarcinoma HeLa cells used in this study were irradiated with a single fraction of GR at various doses $(0,2,8,16,32$ and $64 \mathrm{~Gy})$ and investigated after five time periods $(0 \mathrm{~h}, 24 \mathrm{~h}, 48 \mathrm{~h}$, 60 and72h). The proliferation of HeLa cells was measured by MTT assay as described previously [7]. AI and apoptotic morphological features were assessed by fluorescent microscopy using DAPI staining as described previously [8]. To evaluate mRNA expression in irradiated cells total RNA was extracted using High Pure RNA Isolation kit (Roche) according to the manufacturer's instructions. RNA concentrations were measured with visible spectrophotometer (GBC Cintra 20). After that Human HT-12 Expression Bead Chips with the Illumina Whole-Genome Gene Expression Direct Hybridization Assay (Illumina) system were used to evaluate the expression patterns of more than 48,000 transcripts in HeLa cells, according to previously described [9].

\section{Results and Discussion}

The results of this study after the treatment of a cervical cancer HeLa cells with 2, 8, 16, 32 and 64 Gy of GR for a duration of 0 and 24 hours indicated that radiation inhibits HeLa cells proliferation and apoptosis was raised by time and GR dose rate.

\subsection{GR Inhibits Cell Proliferation}

The survival rates of HeLa cells irradiated by different doses of GR determined by MTT essay. Results showed that radiation inhibits proliferation of HeLa cells proportionally with dose and time after exposure to GR. 32Gy radiation doses induced HeLa cell death by $51 \%$ at 24 hours post-irradiation time compared with control group (not-irradiated). So, 32 Gy of GR dose was determined as the half maximal inhibitory concentration (IC50) dose (Figure 1). In this current study, HeLa cell proliferation was depressed by high doses of GR at 24 hours post-irradiation time. These results are consistent with those of previous studies [10]. 


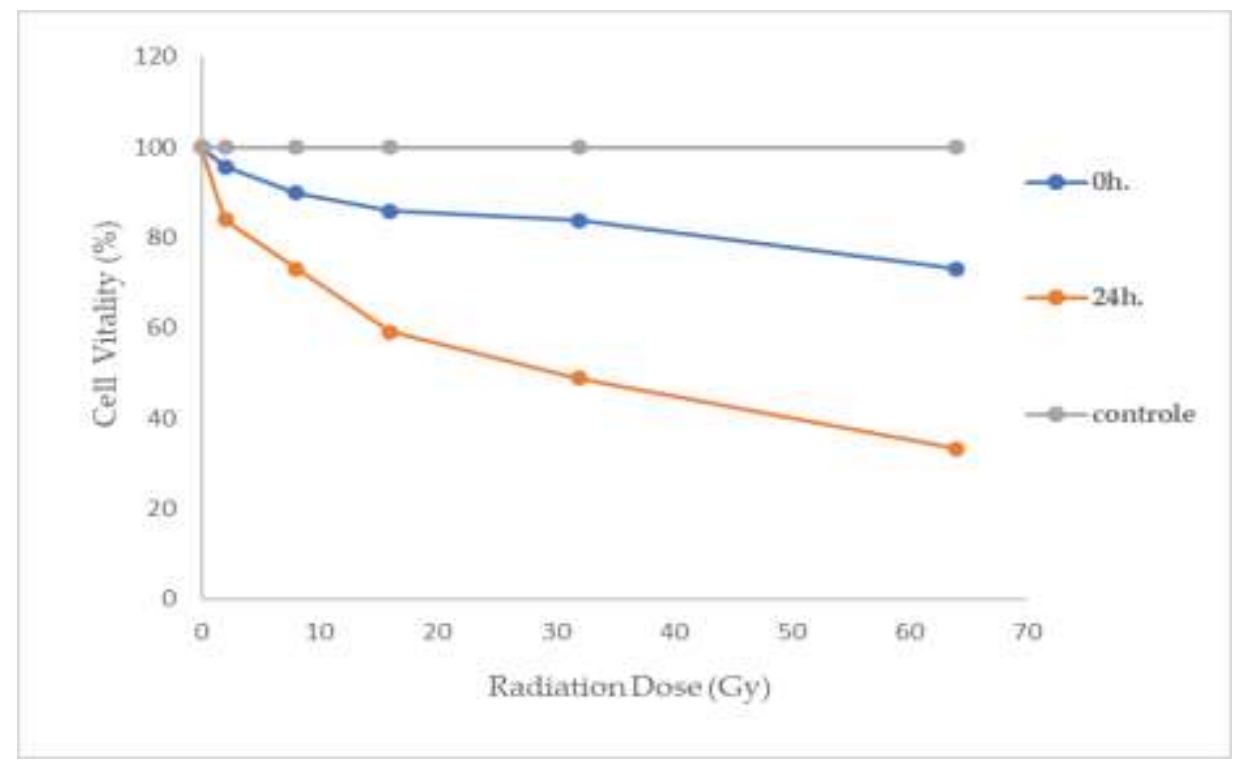

Figure 1. Effect of GR on HeLa cells survival at 0- and 24-hours post-irradiation time using MTT essay. The experiment was repeated triple with similar results.

\subsection{Apoptosis}

Results shows that apoptotic morphological features, such as chromatin condensation, nuclear fragmentation, cell shrinkage and apoptotic bodies formation, was clearly visible under the florescent microscope for gamma irradiated HeLa cells; whereas, the cellular nuclear chromatin in control group homogeneously distribution (Figure 2).

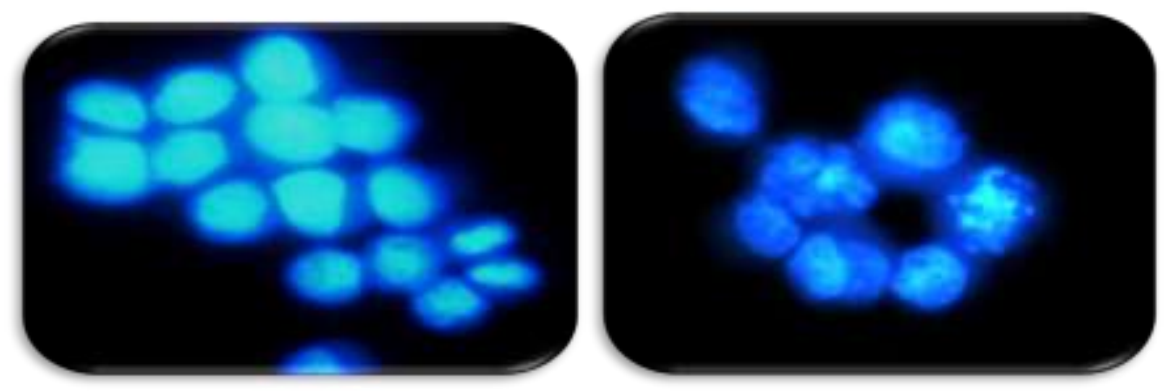

(a)
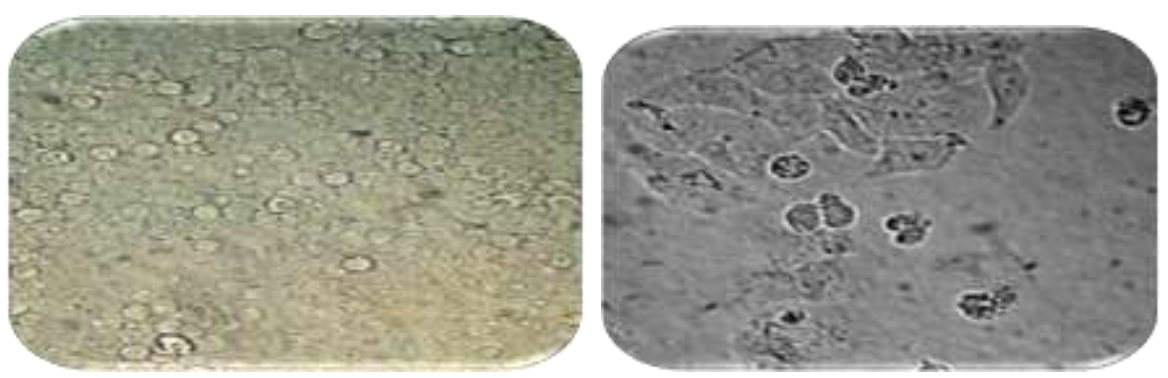

(b)

Figure 2. (a) Representative fluorescent images (X1000) of the nuclear morphology of HeLa cells in the control (left) and 32Gy irradiated groups at 48 hours post-irradiation time following DAPI staining; (b) Description of control (left) and irradiated (right) HeLa cells appearance under the phase contrast microscope after 48 hours incubation (X100).

AI induced by GR after 48 hours exposure to different doses of GR, 32 Gy was specified as an AI dose. These results show that apoptosis induced by gamma radiation and apoptotic morphological 
features, such as chromatin condensation, nuclear fragmentation, cell shrinkage and apoptotic bodies formation, was clearly visible under the florescent microscope for gamma irradiated HeLa cells. after 48 hours exposure to different doses of GR, the dose of 32 Gy was specified as an AI dose. These results indicate that GR primarily decreases HeLa cells proliferation by inducting apoptosis. These results are consistent with those of previous studies [11].

\subsection{Gene Expression and Pathway Analysis}

For statistical analysis of microarray data, the study began with 47,231 probes, which filtered to 47,071 probes. As a result of microarray data analysis, 305 significant genes were selected from HeLa cells irradiated by GR compared with control group. Significant up-regulation of 131 genes and down-regulation of 174 genes were determined.

In this study, pathway analyses were performed using PANTHER on up- and down-regulated genes. The 28 pathways with the best results are listed. Of these, significant upregulation was found in P53, WNT, PDGF, oxidative stress response, interleukin, chemokine and cytokine signalling pathways. The gene members of these signalling pathways MYC, ITPR1 and PERP genes were found to be significantly up-regulated in HeLa cells treated with GR compared to control group of HeLa cells.

These results indicated that PERP expression triggers the death of HeLa cells through the P53-dependent apoptotic pathway. These results agree with other study [5], which showed that PERP was originally identified as a new candidate effector in the P53-dependent apoptotic pathway. As the transcriptional target gene of p53, it is not induced during cell cycle arrest but during apoptosis. With the expression of PERP, induction of apoptosis has been described in fibroblasts, thymocytes and neurons.

\section{Conclusion}

DNA damage signalling network are not the only mediators of apoptosis in response to ionized radiation. Results of this study provide evidence that GR decreases survival of cervical carcinoma HeLa cells by altering the expression of some genes associated with cell proliferation and apoptosis. It was shown that PERP expression triggers the death of HeLa cells through the P53-dependent apoptotic pathway. There is now a very large body of evidence supporting a role for membrane-induced apoptosis in the response to ionized radiation at least in some cells. The major challenges now are to clarify how these pathways progressing.

Author Contributions: “Conceptualization, Khalilia, W. and Özcan, G.; methodology, Khalilia, W. and Özcan, G.; software, Khalilia, W.; validation, Khalilia, W. and Özcan, G.; formal analysis, Khalilia, W. and Özcan, G.; investigation, Özcan, G.; resources, Khalilia, W. and Özcan, G.; data curation, Özcan, G.; writing-original draft preparation, Khalilia, W.; writing-review and editing Khalilia, W. and Özcan, G.; visualization, Khalilia, W. and Özcan, G.; supervision, Özcan, G.; project administration, Khalilia, W. and Özcan, G..; funding acquisition, Khalilia, W. and Özcan, G.; All authors have read and agreed to the published version of the manuscript."

Funding: “This research was funded by Scientific Research Projects Coordination Unit of Istanbul University., grant number 46813"

Conflicts of Interest: "The authors declare no conflict of interest."

\section{References}

1. Wyllie, A.H. A Brief Review of Apoptosis Biology. Mol. Neurobiol. 2010, 42, 4-9, doi:10.1007/s12035-010-81255

2. Cui, Q.; Yu, J.H.; Wu, J.N.; Tashiro, S.; Onodera, S.; Minami, M.; et al. P53- mediated cell cycle arrest and apoptosis through a caspase-3- independent, but caspase-9-dependent pathway in oridonin-treated MCF-7 human breast... Acta Pharmacol Sin. 2007; 28: 1057-1066

3. Li, B.; Zhao, J.; Wang, C.Z.; Searle, J.; He, T.C.; Yuan, C.; et al. Ginsenoside Rh2 induces apoptosis and paraptosis-like cell death in colorectal cancer cells through activation of p53. Cancer Lett. 2011; 301: 185-192.

4. Attardi, L.D.; Reczek, E.E.; Cosmas, C.; Demicco, E.G.; McCurrach, M.E.; Lowe, S.W.; \& Jacks, T. PERP, an apoptosis-associated target of p53, is a novel member of the PMP-22/gas3 family. Genes \& Development. 
2000, 14(6), 704-718.

5. Davies, L., Gray, D., Spiller, D., White, M. R. H., Damato, B., Grierson, I., \& Paraoan, L. P53 apoptosis mediator PERP: localization, function and caspase activation in uveal melanoma. Journal of Cellular and Molecular Medicine. 2009, 13(8b), 1995-2007. http://doi.org/10.1111/j.1582-4934.2008.00590.x

6. Paraoan, L.; Gray, D.; Hiscott, P.; Ebrahimi, B.; Damato, B.; \& Grierson, I. Expression of p53-induced apoptosis effector PERP in primary uveal melanomas: downregulation is associated with aggressive type. Experimental Eye Research. 2006, 83(4), 911-919. http://doi.org/10.1016/j.exer.2006.04.016

7. Ö̈zcan, G.; Dağdeviren, Ö.; Akman, G.; Khalilia, W.; Tezel Yetiz, B.; Karagöz, A.; Melikoglu, G.; Anil, S.; Kultur, S.; Sutlupinar, N. Screening for antitumor activity of various plant extracts on HeLa and C 4-I cell lines. J. Buon 2016, 21, 1552-1560.

8. Khalilia, W. Induction of Apoptosis Pathways in Human Cervical Carcinoma Cells by Ionizing Radiation. In Recent Research Advances in Biology, $4^{\text {th }}$ ed., Özcan, G.; bookpi: Online, 2020, Volume 4, pp. 10-20. https://doi.org/10.9734/bpi/rrab/v4

9. Khalilia, W.M.; Özcan, G.; Karaçam, S. Gene Expression and Pathway Analysis of Radiation-Induced Apoptosis in C-4 I Cervical Cancer Cells. J. Autoimmun. Res. 2017, 4, 1014

10. Bian, J.; Wang, X.; Yun, J.; Cao, R.; Cao, Y.; Liang, J.; Ma, B.F. Single-fraction $\gamma-60$ Co radiation induces apoptosis in cultured rat C6 cells. Ann. Saudi Med. 2012, 32, 269-275

11. Eriksson, D.; Löfroth, P.; Johansson, L.; Riklund, A.K.; Stgbrand, T. Apoptotic signalling in HeLa Hep2 cells following 5 Gy of Cobalt-60 gamma radiation. Anticancer Research 2009, 29, 4361-4366

(C) 2020 by the authors. Submitted for possible open access publication under the terms and conditions of the Creative Commons Attribution (CC BY) license (http://creativecommons.org/licenses/by/4.0/). 